\title{
Acute myeloid leukemia in a young male patient with Behçet's disease presenting with febrile neutropenia
}

\author{
Ashish Sharma ${ }^{1}$, Mohammad Ali $^{2}$, Shruti Chauhan ${ }^{3}$, Smita Singh $^{3}$, Vivek Arya ${ }^{4}$ \\ ${ }^{1}$ Department of Rheumatology, Fortis Flt. Lt. Rajan Dhall Hospital, New Delhi, India \\ 2Department of Medicine, Dr. Ram Manohar Lohia Hospital and Post Graduate Institute of Medical Education and Research, \\ New Delhi, India \\ ${ }^{3}$ Department of Pathology, Lady Hardinge Medical College, University of Delhi, New Delhi, India \\ ${ }^{4}$ General Medicine, North Devon District Hospital, Barnstaple, United Kingdom
}

\begin{abstract}
Behçet's disease is a multi-systemic vasculitis which is characterized by recurrent oral and genital ulceration with positive pathergy test. These features may also be seen in various hematological malignancies. In patients with leukemia who present with Behçet's disease-like features, it is often difficult to ascertain whether the clinical manifestations are due to leukemia, or there is co-existing Behçet's disease too. The authors report an Indian farmer who presented with clinical features suggestive of Behçet's disease with profound neutropenia. On bone marrow examination he was found to have acute monocytic leukemia (AML-M5). Chemotherapy for leukemia did not relieve his oral and genital ulcers, for which glucocorticoids and colchicine had to be given. So the patient had co-existence of Behçet's disease along with AML-M5, which has never been reported in the literature before.

Key words: acute myeloid leukemia, neutropenia, Behçet’s disease, pathergy reaction.
\end{abstract}

\section{Introduction}

Behçet's disease (BD) is a multi-systemic vasculitis involving both arteries and veins, characterized by recurrent oral and genital ulcers with typical cutaneous and ocular features [1]. There are a few case reports describing the association of malignancies with BD [2]. Among hematological malignancies, chronic myeloid leukemia, lymphoma and rarely acute myeloid leukemia have been reported in patients with $\mathrm{BD}$.

The authors report, to the best of our knowledge, the first case of an association of BD with acute monocytic leukemia (AML-M5).

\section{Case report}

A 35-year-old farmer presented with complaints of swelling of his left lower limb and continuous fever for 1 week. His illness dated back 6 months when he started having recurrent, painful oral ulcers for which he took some topical treatment with which he obtained partial relief; the ulcers, however, continued to appear. A fortnight after the onset of these ulcers, he started having fever which was intermittent. Two months into his illness, he noticed ulcerated, painful lesions over his penis and scrotum which did not heal (Figs. 1 and 2).

One week before presenting to our hospital, the patient developed swelling of his left lower limb without any preceding history of prolonged immobilization, surgery or trauma; his fever by now became high grade $\left(104.8^{\circ} \mathrm{F}=40.4^{\circ} \mathrm{C}\right)$ and continuous nature.

Besides recurrent oral and genital ulcers, there was no suggestion of any focus of infection which could explain his fever. He was married and had 5 children; there was no history of urethral discharge or of sexual promiscuity. The patient did not give a history of skin rashes. He had no visual complaints.

On examination, the patient was pale, his pulse rate was $120 /$ minute, temperature $38^{\circ} \mathrm{C}$, respiratory rate $20 / \mathrm{min}$ -

Address for correspondence:

Ashish Sharma, Department of Rheumatology, Fortis Flt. Lt. Rajan Dhall Hospital, Aruna Asaf Ali Marg, Pocket 1, Sector B, Vasant Kunj, 110070 New Delhi, India, e-mail: ash.blueney@gmail.com

Submitted: 8.02.2019; Accepted: 3.06.2019 


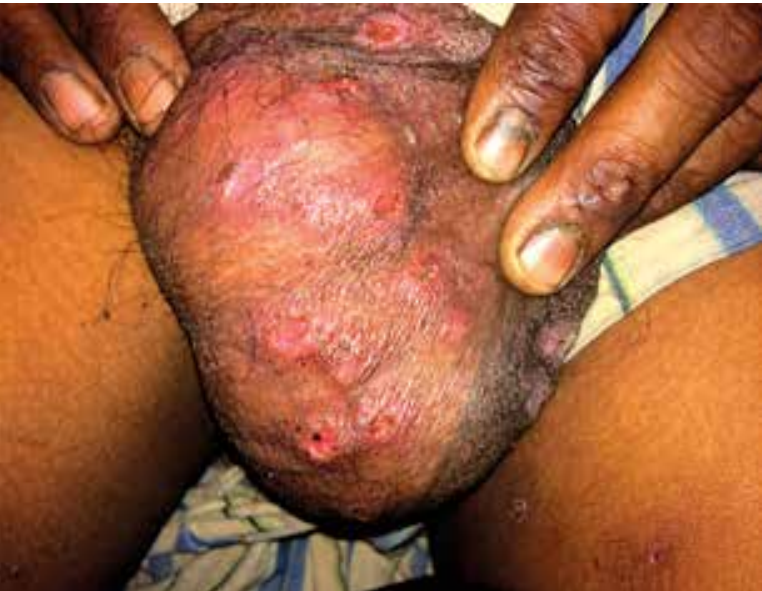

Fig. 1. Sharply demarcated scrotal ulcers with surrounding erythema.

ute and blood pressure 110/70 mm Hg. There was marked swelling and induration of the left leg and thigh without any local warmth. There was a $3 \mathrm{~mm}$, tender ulcer on the hard palate, with a clean base and erythematous margins. Abdominal examination revealed a firm, enlarged, smooth liver, extending $2 \mathrm{~cm}$ below the right costal margin. There were sharply defined, punched out, tender ulcers over the scrotal skin, 3 to $5 \mathrm{~mm}$ in diameter, with a clean base and surrounding erythema (Fig. 1). A similar ulcer was present on the glans penis (Fig. 2).

Laboratory evaluation revealed an increased erythrocyte sedimentation rate (ESR) of $55 \mathrm{~mm}$ in the first hour, decreased serum concentration of hemoglobin $6.2 \mathrm{~g} / \mathrm{dl}$, white blood cell count (WBC) 910 cells $/ \mathrm{mm}^{3}$, absolute neutrophil count 270 cells $/ \mathrm{mm}^{3}$ and platelet count (PLT) 172,000 cells $/ \mathrm{mm}^{3}$. Peripheral blood smear examination showed leucopenia with normocytic normochromic anemia with mild anisocytosis and occasional blasts. Enzyme-linked immunosorbent assays (ELISA) for HIV detection was non-reactive and the venereal disease research laboratory test (VDRL), Treponema pallidum hemagglutination (TPHA) test and rapid plasma reagin test (RPR) were negative.

A venous Doppler examination of the left lower limb showed thrombosis extending from the left popliteal vein to the left common iliac vein. The coagulation profile was normal. Hepatitis B surface antigen ( $\mathrm{HBsAg}$ ) was not detected and antibodies to the hepatitis $C$ virus were absent. The combination of recurrent oral and genital ulceration with deep vein thrombosis suggested the diagnosis of BD; the pathergy test was positive.

Although neutropenia and anemia have occasionally been reported in BD, the marked leucopenia led us to perform a bone marrow examination, which revealed $65 \%$ blasts and promonocytes (Fig. 3). Flow cytometry

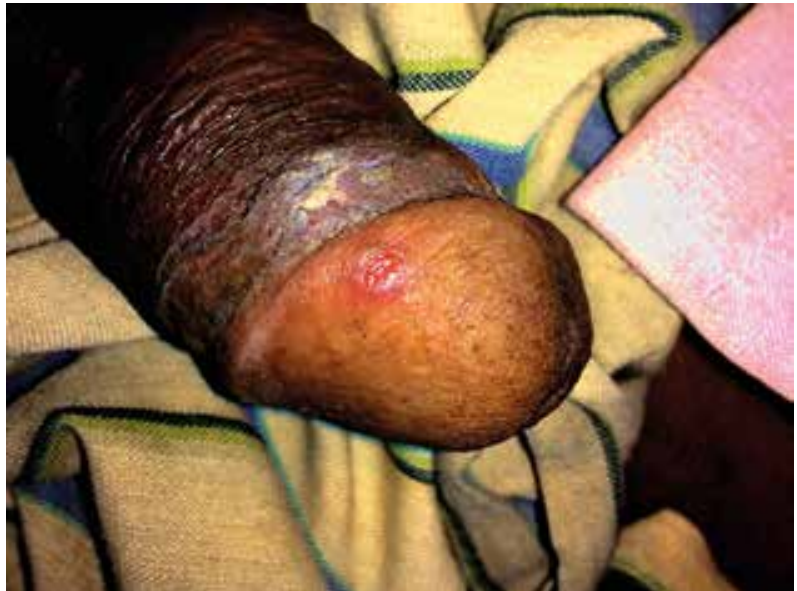

Fig. 2. Ulcer on glans penis.

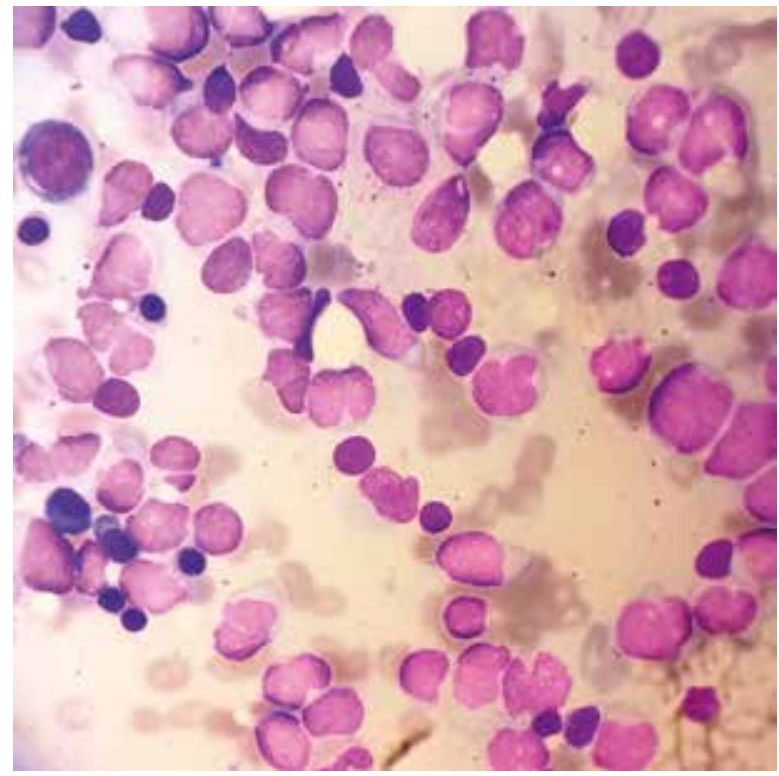

Fig. 3. Bone marrow aspirate showing an expanded marrow with blasts and promonocytes having convoluted nuclei and lacy chromatin.

showed positivity for CD 33, CD 117, CD 34, CD 11b and CD 11c. These findings suggested a diagnosis of acute monocytic leukemia - AML-M5 according to the WHO classification. The patient was started on broad spectrum intravenous antibiotics for febrile neutropenia and was referred to a hematological department for further management of leukemia. Although the blood parameters improved with chemotherapy, oral and genital ulcers continued to occur. $0.5 \mathrm{mg} / \mathrm{kg}$ prednisolone (in tapering doses) and colchicine were also added to the treatment with a positive effect and significant clinical improvement. 


\section{Discussion}

Behçet's disease is a multi-systemic vasculitis involving both arteries and veins, characterized by recurrent oral and genital ulcers [1]. Deep vein thrombosis occurs in about $39 \%$ of patients with BD [2]. Our patient fulfilled the criteria of the International Study Group for Behçet's disease, as he had recurrent oral and genital ulcers with a positive pathergy test and in addition had lower limb deep vein thrombosis [1].

Neutropenia has occasionally been reported to be due to BD itself. However, bone marrow failure secondary to associated myelodysplastic syndrome (MDS) is more often the cause of leucopenia in $\operatorname{BD}[3,4]$. Trisomy of chromosome 8 is commonly seen in patients with co-existence of BD and MDS. Mantzourani et al. [5] reported a rare occurrence of chronic myelomonocytic leukemia with MDS in a patient with BD and trisomy of chromosome 8. In our patient, profound leucopenia with febrile neutropenia and anemia prompted a bone marrow examination which revealed features diagnostic of acute myeloid leukemia.

Behçet's disease is a state of chronic inflammation as evidenced by increased expression of inflammatory markers, which can lead to malignant transformation of cells in various tissues, including hematopoietic cells [6]. Association of malignancies with BD has been reported in the literature [7]. Among hematological malignancies, chronic myeloid leukemia and lymphoma have been reported in patients with BD [8]. In only a few cases has acute myeloid leukemia been found in patients with Behçet's disease [9-11]. A large study on 651 patients with $\mathrm{BD}$ showed that malignancy occurred in $6.3 \%$ of the patients [9]. Out of these, hematological malignancies were present in $70.7 \%(n=29)$ of cases. Of these 29 cases, AML was found in 5 patients. To the best of our knowledge, this is the first reported case of BD with acute monocytic leukemia (AML-M5).

As there is no confirmatory test for BD, the diagnosis is made on clinical grounds. Clinical diagnosis of BD ultimately turning out to be leukemia has been reported in the literature, since many of the clinical features of BD may also be seen in leukemia (including the pathergy test) [12-14].

Koba et al. [14] reported a case of a 74-year-old female patient who had fever, oral ulcers, acne-form skin lesions and ulcerations in ileum and colon which suggested the diagnosis of BD with intestinal involvement. The described patient developed monocytosis and bone marrow examination revealed AML-M5. The authors concluded that the features suggestive for Behçet's disease were misleading, and hematological malignancy was the final diagnosis. It is often difficult to ascertain whether a leukemic patient is suffering from BD in addition, as in our patient.

Treatment needs to be tailored to the clinical manifestations of BD. Systemic glucocorticosteroids, azathioprine and colchicine are effective for many of the manifestations of this disease. Tumor necrosis factor inhibitors (TNFi) have also been found to be effective [15]. Hematopoietic stem cell transplant (HSCT) is considered curative for acute myeloid leukemia. HSCT has also been shown to be very effective in inducing remission in Behçet's disease $[11,16]$.

\section{Conclusions}

In the presence of characteristic clinical features such as recurrent oral and genital ulceration and deep vein thrombosis, it is not difficult to diagnose BD. However, our patient's marked cytopenia drew attention to a more serious second diagnosis - acute myeloid leukemia. His oral and genital ulcers did not respond to chemotherapy. Addition of prednisolone and colchicine led to a marked improvement. Published literature has evidence of co-existence of BD and AML, as well as of leukemia masquerading as BD. This clinical scenario remains a challenge and perhaps the degree of response to anti-leukemic therapy in a particular patient will help resolve this dilemma.

The authors declare no conflict of interest.

\section{References}

1. International Study Group for Behçet's disease. Criteria for diagnosis of Behçet's disease. Lancet 1990; 335: 1078-1080.

2. Houman M, Ghorbel I, Salah I, et al. Deep vein thrombosis in Behçet's disease. Clin Exp Rheumatol 2001; 19: S48-S50.

3. Leonard RCF, Thompson RB. Behçet's syndrome and neutropenia. Postgrad Med J 1981; 57: 448-449.

4. Oh E, Yoon J, Park Y, et al. Behcet's disease associated with myelodysplastic syndrome: a case report. J Korean Med Sci 1999; 14: 685-687.

5. Mantzourani MG, Chantziara K, Thanopoulou I, et al. Coexistence of Behçet's disease and chronic myelomonocytic leukemia with trisomy 8: a case report and review of literature. Clin Exp Rheumatol 2009; 27: S85-87.

6. Budak-Alpdoğan T, Demirçay Z, Alpdoğan O, et al. Behçet's disease in patients with chronic myelogenous leukemia: possible role of interferon-alpha treatment in the occurrence of Behçet's symptoms. Ann Hematol 1997; 74: 45-48.

7. Cengiz M, Altundag M, Zorlu A, et al. Malignancy in Behçet's disease: $A$ report of 13 cases and a review of the literature: Clin Rheumatol 2001; 20: 239-244.

8. Houman M, Ben I, B'Chir-Hamzaoui S, et al. Lymphome intestinal associé à une maladie de Behçet. Ann Med Interne 2001; 152: 415-418. 
9. Lin Y, Li G, Zheng W, et al. Behcet's disease associated with malignancy: a report of 41 Chinese cases. Int J Rheum Dis 2014; 17: 459-465.

10. Kaloterakis A, Stavrianeas N, Karagianni I, et al. Adamantiades-Behçet's disease coexisting with acute myeloblastic leukaemia. Br J Dermatol 1997; 137: 317-318.

11. Nakamura Y, Matsuguma M, Tokunaga, Y, et al. Successful treatment of Behçet's disease associated with acute myeloid leukemia with myelodysplasia-related changes using azacitidine and tacrolimus before allogenic hematopoietic stem cell transplantation. Intern Med 2017; 56: 1199-1202.

12. Balasooriya B, Fonseka H, Williams S, et al. Oro-genital ulcers with a positive pathergy test in acute myeloid leukaemia. Ceylon Med J 2009; 54: 131-132.
13. Oksuz M, Coskun B, Tufan A, et al Hairy cell leukemia presenting initially with symptoms of Behçet's disease. Int J Rheum Dis 2014; 17: 689-692.

14. Koba S, Sekioka T, Takeda S, et al. Acute Monocytic Leukemia Masquerading Behçet's Disease-Like Illness at Onset in an Elderly Female. Case Rep Hematol 2016: 2016: 4231276.

15. Vallet $H$, Riviere S, Sanna A, et al. Efficacy of anti-TNF alpha in severe and/or refractory Behçet's disease: Multicenter study of 124 patients. J Autoimmun 2015; 62: 67-74.

16. Lim S, Hulsey M, Esler W. Resolution of Behçet's disease after non-myeloablative allogeneic stem cell transplant for acute myeloid leukaemia. Rheumatology 2009; 48: 88-89. 